\title{
A simple model for dynamical melting of moving vortex lattices interacting with periodic pinning
}

\author{
Clécio C. de Souza Silva \\ Departamento de Física, Universidade Federal de Pernambuco 50670-901, Recife-PE, Brasil \\ Gilson Carneiro* \\ Instituto de Física, Universidade Federal do Rio de Janeiro, C.P. 68528, 21945-970, Rio de Janeiro-RJ, Brasil
}

(November 4, 2018)

\begin{abstract}
Melting of moving vortex lattices in clean superconducting films with periodic pinning is studied by a dynamical "cage" model, based on a mean-field treatment of Langevin's equations for the whole vortex lattice, assuming elastic flow. In the frame moving with the velocity of the vortex lattice center of mass, the model describes forced vibrations of a single vortex tied to a spring. The vortex displacements due to thermal fluctuations and to the periodic pinning force, and the relationship between the driving force and the vortex velocity (V-I curves) are obtained by a perturbation method, valid for high velocities and for both weak and strong periodic pinning. The dynamical melting temperature is calculated as a function of the vortex velocity using Lindemann's criterion. Application to a square defect lattice gives, for strong pinning, dynamical melting lines and anisotropic V-I curves that agree qualitatively with numerical results.
\end{abstract}

74.60.Ge, 74.60.Jg

\section{INTRODUCTION}

The study of dynamical phases of lattice structures moving in a periodic medium has received a great deal of attention lately due to its relevance to physical systems such as adsorbed atomic layers in boundary dubricationt, vortices in Josephson Junction Arrays (JJA) 2 and in films with artificial defect lattices.

Numerical studies of driven vortices in clean superconducting films interacting with aperiodic lattice of columnar defects (CD) of a moving vortex-lattice (VL) into a moving vortex liquid. In these systems the moving vortices order in a VL at low temperatures when the driving force magnitude is sufficiently large. Melting of the VL is found to take place at a temperature that, for a given vortex density, decreases with the driving force magnitude, approaching the equilibrium VL melting temperature when this magnitude approaches infinity. These studies also show that the vortices center of mass $(\mathrm{CM})$ velocity is in general not parallel to the driving force. This leads to anisotropic VI curves, with voltages both longitudinal and transverse to the direction of the applied current.

In this paper we show that dynamical melting lines and anisotropic V-I curves similar to those found in the above mentioned numerical studies can be obtained using a simple model for the vortex dynamics.

In driven vortex systems interacting with random or periodic pinning, moving vortex lattices are expected to occur at low temperatures and in the limit of very large $\mathrm{CM}$ velocities, because the pinning potential is averaged in the direction of motion, as first pointed out by Schmid and Hauger 6 . For periodic pinning, Ref. 4 shows that in this limit the moving vortex lattice can be commensurate or incommensurate with the periodic pinning potential, depending on the direction of motion and on the vortex density. Commensurate lattices occur only for motion along directions of high symmetry of the pinning potential, such as $[1,0],[0,1],[1,1]$ and $[-1,1]$ for soure symmetry, if the vortex density is not too high 49 . to. For motion along other directions, the moving vortex lattices are incommensurate. Recent numerical simulations find that both moving commensurate and incommensurate lattices y undergo dynamical melting into a moving liquide 610. However, dynamical melting of commensurate lattices is much more complicated than that of the incommensurate ones. The reason is that the moving commensurate lattices are pinned in channels running along the direction of motion (transverse pinning), and melting into a liquid occirs simultaneously with depinning from the channels 4 . 10 . In this paper we consider only dynamical melting of incommensurate lattices.

Dynamical melting of vortex lattices is still poorly understood. It is unclear from the simulation results reported so far if the change from lattice to liquid spatial symmetries is a true phase transition, with accompanying singularities in correlation functions, or merely a crossover. In this paper we do not offer any insight into this fundamental question. Our aim is to estimate the dynamic melting temperature based on a well know method for the equilibrium melting temperature, namely Lindemann's criterion. This method was proposed in a classical paper by Houghton, Pelcovitz and Sudbø 1ㅡ. They estimate the equilibrium VL melting temperature for clean bulk superconductors by applying Lindemann's criterion to the mean-square vortex-line displacement caused by thermal fluctuations. A simple model to estimate the 
same temperature was proposed later by Frey, Nelson and Fisher 22. It considers a single vortex line trapped in a harmonic potential representing its interaction with the other vortex lines in the lattice, assumed straight and fixed at their equilibrium lattice positions. We refer to this model here as the equilibrium cage model. The equilibrium melting temperature is estimated by applying Lindemann's criterion to the mean-square thermal fluctuations of the caged vortex line. The result agrees with that of Ref. 11, if the harmonic potential is properly chosen 12. This simple idea can also be applied to twodimensional VL melting in thin films. In this case the equilibrium cage model consists of a point vortex tied to a spring, representing its interaction with the other point vortices, assumed fixed at their lattice positions. The melting temperature is obtained by applying Lindemann's criterion to the caged vortex mean square displacement due to thermal fluctuations 12 . The resulting melting temperature agrees with the one obtained from dislocation unbinding theory 13 , if the spring constant is properly chosen. Our model, which we call the dynamical cage model, generalizes the two-dimensional equilibrium one for a moving vortex lattice. The dynamical melting temperature is estimated by applying Lindemann's criterion to the mean-square vortex displacements caused by thermal fluctuations and by motion in the periodic pinning potential. Application of Lindemann's criterion to estimate the dynamical melting line of vortices in bulk superconductors interacting with random pinning was first proposed by Koshelev and Vinokur 14 .

We start from Langevin's equations describing twodimensional vortices at temperature $T$, interacting between themselves and with a periodic pinning potential, and driven by an external force. We consider vortex motion at large CM velocities and make the following assumptions: i) The moving vortices order in a lattice, incommensurate with the periodic pinning potential. ii) The vortex lattice flows elastically. This means that there are no dislocations and that vortex-vortex interactions can be approximated by harmonic forces. We then propose a mean-field like treatment of the resulting equations of motion. This approximation reduces the problem to that of a single vortex tied to a spring, both moving with the $\mathrm{CM}$ velocity, and interacting with the periodic pinning potential. We find that the dynamical cage model is governed by two equations of motion. One for the vortex displacement in the frame moving with the vortex lattice CM velocity (CM frame), and another relating the driving force and the CM velocity. The vortex displacement equation is found to be nonlinear, unlike that for the equilibrium cage model, due to the vortexpinning interaction. These equations are solved by a perturbation method, similar to that introduced by Schmid and Hauger $\mathrm{G}$ for weak random pinning forces. It consists in expanding the time-dependent periodic pinning force (in the CM frame) in powers of the vortex displacement. We find that the perturbation expansion is valid at large $\mathrm{CM}$ velocities for both weak and strong pinning. From these solutions we obtain expressions for the vortex mean square displacement and for the relationship between the CM velocity and the driving force. The dynamical melting temperature is obtained by applying Lindemann's criterion to the caged vortex mean-square displacement. These results are applied to a square pinning array, and compared to numerical simulation ones.

This paper is organized as follows. In Sec. II we derive the dynamical cage model equations of motion and their perturbation theory solution. In Sec. III. we apply these results to strong pinning by a square array of pinning centers. The conclusions of the paper are presented in Sec. IV. Details of the perturbation calculation are given in the Appendix.

\section{DYNAMICAL CAGE MODEL}

We consider $N_{v}$ two-dimensional vortices at temperature $T$ interacting between themselves and with a periodic potential produced by an array of pinning centers and driven by a force $\mathbf{f}_{d}$. The equations of motion are 15

$$
\eta \frac{d \mathbf{r}_{j}}{d t}=\mathbf{f}_{d}+\mathbf{F}_{j}^{v-v}+\mathbf{F}^{v-p}\left(\mathbf{r}_{j}\right)+\boldsymbol{\Gamma}_{j},
$$

where $j=1, \ldots, N_{v}, \eta$ is the friction coefficient,

$$
\mathbf{F}_{j}^{v-v}=-\sum_{i \neq j=1}^{N_{v}} \nabla_{j} U^{v-v}\left(\mathbf{r}_{j}-\mathbf{r}_{i}\right)
$$

is the force of interaction with other vortices, $U^{v-v}(\mathbf{r})$ being the vortex-vortex interaction potential in two dimensions,

$$
\mathbf{F}^{v-p}\left(\mathbf{r}_{j}\right)=\sum_{\mathbf{Q}}(-i \mathbf{Q}) U_{\mathbf{Q}}^{v-p} e^{i \mathbf{Q} \cdot \mathbf{r}_{j}}
$$

is the force of interaction with the periodic pinning array, $\mathbf{Q}$ denotes the pinning array reciprocal lattice vectors, and $U_{\mathbf{Q}}^{v-p}$ is the Fourier transform of the vortex-single pin interaction potential, and $\boldsymbol{\Gamma}_{j}$ is the random force, satisfying

$$
\left\langle\Gamma_{j \alpha}(t) \Gamma_{l \beta}\left(t^{\prime}\right)\right\rangle=2 k_{B} T \eta \delta_{j, l} \delta_{\alpha, \beta} \delta\left(t-t^{\prime}\right),
$$

where $\langle\cdots\rangle$ denotes average over the random force distribution.

We assume that the vortices center of mass (CM) moves with constant velocity $\mathbf{v}$, defined, as usual, by

$$
\mathbf{v}=\frac{1}{N_{v}} \sum_{j=1}^{N_{v}} \frac{d \mathbf{r}_{j}(t)}{d t}
$$

Using Eq. (11), we find that

$$
\eta \mathbf{v}=\mathbf{f}_{d}+\frac{1}{N_{v}} \sum_{j=1}^{N_{v}} \mathbf{F}^{v-p}\left(\mathbf{r}_{j}(t)\right) .
$$


To derive Eq. (6) we use the fact that for large $N_{v}$, the random force term is negligible, since $\sum_{i=1}^{N_{v}} \boldsymbol{\Gamma}_{j} \sim \sqrt{N_{v}}$. According to Eq. (6), in order that $\mathbf{v}$ is constant $\mathbf{f}_{d}$ must depend on time as well as on the random force. To obtain physical results we average Eq. (6) over time and over the random force distribution, that is

$$
\eta \mathbf{v}=\mathbf{F}_{d}+\frac{1}{N_{v}} \sum_{i=1}^{N_{v}} \frac{1}{\tau} \int_{0}^{\tau}\left\langle\mathbf{F}^{v-p}\left(\mathbf{r}_{j}(t)\right)\right\rangle,
$$

where $\mathbf{F}_{d}$, a constant vector, denotes the average of $\mathbf{f}_{d}$ over time and over the random force distribution and $\tau$ is a time large compared with the characteristic times in Eq. (1). We interpret $\mathbf{F}_{d}$ as the force due to the applied current, so that Eqs. (7) give the V-I curves.

Now we consider a vortex-lattice flowing elastically and transform to the CM frame. We write the vortex positions as

$$
\mathbf{r}_{j}(t)=\mathbf{R}_{j}+\mathbf{v} t+\mathbf{u}_{j}(t),
$$

where $\mathbf{R}_{j}$ and $\mathbf{u}_{j}(t)\left(j=1, \ldots, N_{v}\right)$ are, respectively, the vortex equilibrium positions and displacements from equilibrium in the CM frame. In elastic flow the displacements $\mathbf{u}_{j}(t)$ are small and the vortex-vortex interactions can be approximated by harmonic forces. Our dynamical cage model considers a particular vortex, and substitute its elastic interaction with the other vortices in the lattice by a spring of constant $\kappa$. The equation of motion for this vortex in the CM frame is found to be

$$
\begin{aligned}
\eta \frac{d \mathbf{u}_{l}(t)}{d t}= & -\kappa \mathbf{u}_{l}(t)+\mathbf{F}^{v-p}\left(\mathbf{R}_{l}+\mathbf{u}_{l}(t)+\mathbf{v} t\right) \\
& +\mathbf{f}_{d}-\eta \mathbf{v}+\mathbf{\Gamma}_{l} .
\end{aligned}
$$

The relationship between the spring constant and the vortex-vortex interaction potential will be presented shortly. Our dynamical cage model approach is similar to the mean-field theory introduced by Fisher 16 for sliding charge-density waves interacting with random pinning.

Our objective here is to use Eq. (9), together with Eq. (7), to calculate the vortex mean-square displacement required for Lindemann's criterion, and to obtain the $\mathbf{v}$ vs. $\mathbf{F}_{d}$ relationship (V-I curves). First we note that Eq. (9) depends on the particular vortex $l$, through the $\mathbf{F}^{v-p_{-}}$ term. Since $\mathbf{F}^{v-p}$ is periodic in the pinning-array lattice, its argument in Eq. (9) can be reduced, at any instant $t$, to a position within the pinning-array primitive unit cell. As mentioned in Sec. If we consider only moving incommensurate vortex lattices. In this case the reduced vortex positions are uniformly distributed over the unit cell area. Accordingly, we define the vortex mean square displacement as

$$
u^{2}=\frac{1}{N_{v}} \sum_{j=1}^{N_{v}} \frac{1}{\tau} \int_{0}^{\tau}\left\langle\left|\mathbf{u}_{j}(t)\right|^{2}\right\rangle .
$$

According to the above considerations, our dynamical cage model is described by the set of equations

$$
\begin{aligned}
\eta \frac{d \mathbf{u}_{l}(t)}{d t}= & -\kappa \mathbf{u}_{l}(t)+\mathbf{F}^{v-p}\left(\mathbf{R}_{l}+\mathbf{u}_{l}(t)+\mathbf{v} t\right) \\
& -\frac{1}{N_{v}} \sum_{j=1}^{N_{v}} \mathbf{F}^{v-p}\left(\mathbf{R}_{j}+\mathbf{u}_{j}(t)+\mathbf{v} t\right)+\boldsymbol{\Gamma}_{l},
\end{aligned}
$$

and

$$
\eta \mathbf{v}=\mathbf{F}_{d}+\frac{1}{N_{v}} \sum_{j=1}^{N_{v}} \frac{1}{\tau} \int_{0}^{\tau}\left\langle\mathbf{F}^{v-p}\left(\mathbf{R}_{j}+\mathbf{u}_{\mathbf{v}} t\right)\right\rangle .
$$

To obtain $\mathbf{u}_{l}(t)$ we solve Eq. (11) by the perturbation theory method introduced in Ref. 8, which consists in expanding $\mathbf{F}^{v-p}$ in powers of $\mathbf{u}_{i}(t)$ and solving the resulting equations by iteration. We keep only terms to first order. The relationship between $\mathbf{v}$ and $\mathbf{F}_{d}$ are obtained from Eq. (12) by a similar expansion. The details of these calculation are given in the Appendix.

For the mean square displacement we obtain to leading order

$$
\begin{aligned}
u^{2}= & \frac{2 k_{B} T}{\kappa}+\sum_{\mathbf{Q}} \frac{Q^{2}\left|U_{\mathbf{Q}}\right|^{2}}{\eta^{2}(\mathbf{Q} \cdot \mathbf{v})^{2}+\kappa^{2}} \\
& +\frac{2 k_{B} T}{\kappa} \sum_{\mathbf{Q}} \frac{Q^{4}\left|U_{\mathbf{Q}}\right|^{2}}{\eta^{2}(\mathbf{Q} \cdot \mathbf{v})^{2}+4 \kappa^{2}} .
\end{aligned}
$$

To the same order of approximation the $\mathbf{v}$ vs. $\mathbf{F}_{d}$ relationship is

$$
\begin{aligned}
\mathbf{v}= & \frac{\mathbf{F}_{d}}{\eta}-\sum_{\mathbf{Q}} \frac{\mathbf{Q} Q^{2}\left|U_{\mathbf{Q}}\right|^{2}}{\eta^{2}(\mathbf{Q} \cdot \mathbf{v})^{2}+\kappa^{2}} \mathbf{Q} \cdot \mathbf{v} \\
& -\frac{k_{B} T}{\kappa} \sum_{\mathbf{Q}} \frac{\mathbf{Q} Q^{4}\left|U_{\mathbf{Q}}\right|^{2}}{\eta^{2}(\mathbf{Q} \cdot \mathbf{v})^{2}+4 \kappa^{2}} \mathbf{Q} \cdot \mathbf{v} .
\end{aligned}
$$

According to Lindemann's criterion the VL melts when $u^{2}=c_{L}^{2} a_{v}^{2}$, where $a_{v}$ is the VL lattice parameter. Thus, our dynamical cage model only makes sense as long as $u^{2}<c_{L}^{2} a_{v}^{2}$. This requires that in Eq. (13) both $k_{B} T / \kappa$, and the velocity dependent terms are sufficiently small compared to $a_{v}^{2}$. The latter are so for large enough $v$. To state more precisely the conditions under which the perturbation expansion in Eq. (13) is valid, we restrict our considerations to periodic pinning forces that can be represented by Eq. (3) with only a few non-zero $U_{\mathbf{Q}}$. Typically those for $\mathbf{Q}$ corresponding to nearest and nextnearest neighbor reciprocal lattice points. In these cases $Q \sim 2 \pi / a_{p}, a_{p}$ being the pinning array lattice parameter. We also assume that the number of vortices is comparable to the number of pins, so that $a_{v} \sim a_{p}$. Under these assumptions, the condition for the smallness of both velocity dependent terms in Eq. 13 depends on the relative strengths of the pinning and elastic forces. For weak pinning, defined as $\left|U_{\mathbf{Q}}\right| \ll \kappa a_{p}^{2} / 2 \pi$, these terms are always small, independent of $\mathbf{v}$, so that the effects of vortex 
(a)

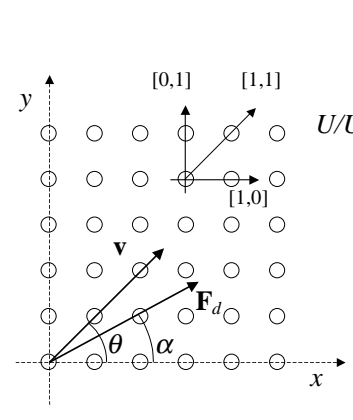

(b)

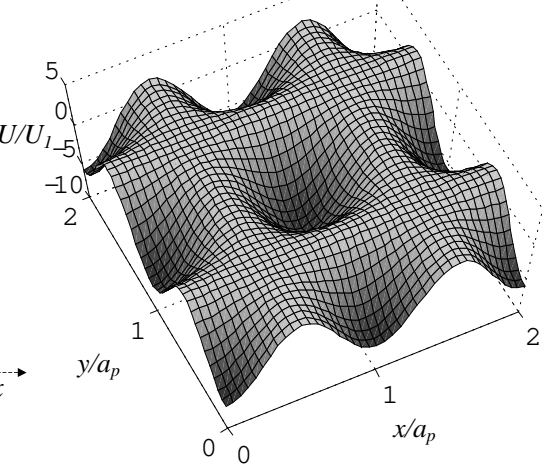

FIG. 1. (a) Pinning lattice, coordinate system and angles defining the directions of the center of mass velocity, $\mathbf{v}$, and the driving force, $\mathbf{F}_{d}$. (b) Square pinning potential (Eq. (17)) with $U_{2}=U_{1} / 2$.

motion on $u^{2}$ are negligible. For strong pinning, that is $\left|U_{\mathbf{Q}}\right| \sim \kappa a_{p}^{2} / 2 \pi$, the velocity dependent terms in Eq. (13) are small for all $\mathbf{Q}$, if $\eta v \cos \vartheta_{\mathbf{Q}} \gg \kappa a_{p} / 2 \pi$, where $\vartheta_{\mathbf{Q}}$ is the angle between the particular $\mathbf{Q}$ and $\mathbf{v}$. Similar considerations apply to the perturbation expansion for $\mathbf{v}$, Eq. (14). For strong pinning, the perturbation expansion breaks down if, for some $\mathbf{Q}$ entering the sums in Eq. (13), $\mathbf{Q} \cdot \mathbf{v}=0\left(\cos \vartheta_{\mathbf{Q}}=0\right)$. In this case the velocity dependent terms in Eq. (13) are not small. This breakdown can also be seen from Eq. (9). If $\mathbf{Q} \cdot \mathbf{v}=0$ the pining force $\mathbf{F}^{v-p}\left(\mathbf{R}_{l}+\mathbf{u}_{l}(t)+\mathbf{v} t\right)$ has a static component, periodic in the direction perpendicular to $\mathbf{v}$, which gives rise to a static vortex displacement comparable to $a_{v}$ for strong pinning. Such a displacement indicates that our assumption that the moving VL is incommensurate with the pinning array lattice breaks down for motion along the directions for which $\mathbf{Q} \cdot \mathbf{v}=0$. There are only a few directions of motion for which $\mathbf{Q} \cdot \mathbf{v}=0$ since, as mentioned above, only a few $\mathbf{Q}$ enter the sums in Eq. (13). We note that the terms in sum in Eq. (14) with $\mathbf{Q} \cdot \mathbf{v}=0$ do not contribute to $\mathbf{v}$, so that there is no breakdown in the perturbation expansion for this quantity.

In the limit of very large $v(v \rightarrow \infty)$ our dynamical cage model reduces to the static one, since vortex motion averages out the pinning potential (we assume $\mathbf{Q} \cdot \mathbf{v} \neq 0$ ). In this limit $u^{2}$ reduces to the equilibrium mean square displacement of a vortex tied to a spring, $u^{2}=\frac{2 k_{B} T}{\kappa}$. Application of Lindemann's criterion gives for the melting temperature $k_{B} T_{m}=\kappa a_{v}^{2} c_{L}^{2} / 2$. To compare this estimate of $T_{m}$ with the dislocation unbinding theory one, $\kappa$ is identified with $\kappa=\left(\partial^{2} U^{v-v}(r) / \partial r^{2}\right)_{r=a_{v}}$, where $U^{v-v}(r)=\left(\phi_{0}^{2} / 8 \pi A\right) \ln (r / \xi)$ is the $2 \mathrm{D}$ vortex-vortex interaction potential12 ( $\phi_{0}$ is the flux quantum and $\Lambda$ the film effective penetration depth ). The result is $\kappa=\phi_{0}^{2} / 8 \pi \Lambda a_{v}^{2}$ and $k_{B} T_{m}=c_{L}^{2} \phi_{0}^{2} / 16 \pi \Lambda$. This melting temperature agrees with the dislocation unbinding the- ory ond 13 if $c_{L} \simeq 0.12$. From here on we adopt for $\kappa$ and $c_{L}$ the above mentioned values.

Application of Lindemann's criterion to Eq. (13) gives the velocity dependent melting temperature $T_{d m}(\mathbf{v})$ as

$$
k_{B} T_{d m}(\mathbf{v})=\frac{\frac{\kappa a_{v}^{2} c_{L}^{2}}{2}-k_{B} T_{p p}(\mathbf{v})}{1+\sum_{\mathbf{Q}} \frac{Q^{4}\left|U_{\mathbf{Q}}\right|^{2}}{\eta^{2}(\mathbf{Q} \cdot \mathbf{v})^{2}+4 \kappa^{2}}},
$$

where

$$
k_{B} T_{p p}(\mathbf{v}) \equiv \frac{\kappa}{2} \sum_{\mathbf{Q}} \frac{Q^{2}\left|U_{\mathbf{Q}}\right|^{2}}{\eta^{2}(\mathbf{Q} \cdot \mathbf{v})^{2}+\kappa^{2}}
$$

is identified with an effective temperature, resulting from vortex vibrations due to the periodic pinning force, that adds to the thermodynamic one in the expression for $u^{2}$. The temperature $T_{p p}$ is similar to the 'shaking temperature' introduced by Koshelev and Vinokur 14 for random pinning. It arises from a $T$-independent contribution to $u^{2}$, the second term in the right hand side of Eq. (13). There is also a $T$-dependent contribution to $u^{2}$ in Eq. (13) that cannot be identified with an effective temperature. However, this term leads to a small correction in $T_{d m}(\mathbf{v})$, since the denominator in Eq. (15) is close to one. Thus, the dynamical melting condition is essentially that $k_{B}\left(T_{d m}(\mathbf{v})+T_{p p}(\mathbf{v})\right)=\kappa a_{v}^{2} c_{L}^{2} / 2$. The $\mathbf{v}$ dependence of $T_{d m}(\mathbf{v})$, Eq. (15), is such that $T_{d m}(\mathbf{v})$ decreases with decreasing $v$.

The V-I curves follow from the relationship between $\mathbf{v}$ and $\mathbf{F}_{d}$, Eq. (14). In the $v \rightarrow \infty$ limit $\mathbf{v}=\mathbf{F}_{d} / \eta$. For finite $v$, vortex motion is no longer along the drive direction, leading to anisotropic $\mathrm{V}-\mathrm{I}$ curves. The $\mathbf{v}$ vs. $\mathbf{F}_{d}$ relationship also allows us to obtain the melting temperature as a function of the driving force, which is more usual.

In the next section we study in detail the dynamical cage model predictions by applying them to a typical periodic pinning potential and carrying out numerical calculations.

\section{SQUARE PINNING-LATTICE}

We consider a square pinning array with lattice parameter $a_{p}$, and assume that the vortex-pinning interaction force is given by Eq. (3) with the $U_{\mathbf{Q}}^{v-p}$ chosen as

$$
U_{\mathbf{Q}}^{v-p}= \begin{cases}U_{1} & \text { for } \mathbf{Q}= \pm \frac{2 \pi}{a_{p}} \hat{x}, \pm \frac{2 \pi}{a_{p}} \hat{y} \\ U_{2} & \text { for } \mathbf{Q}= \pm \frac{2 \pi}{a_{p}}(\hat{x} \pm \hat{y}) \\ 0 & \text { otherwise }\end{cases}
$$

The $x$ and $y$ axis are along the pinning lattice $[1,0],[0,1]$ directions, respectively, as shown in Fig. 1(a). We choose $U_{2}=U_{1} / 2$. This gives the pinning potential shown in Fig. 11(b). We set $U_{1} / \kappa a_{p}^{2}=1 / 2 \pi=0.16$. As discussed in Sec. II, this value corresponds to strong pinning. 

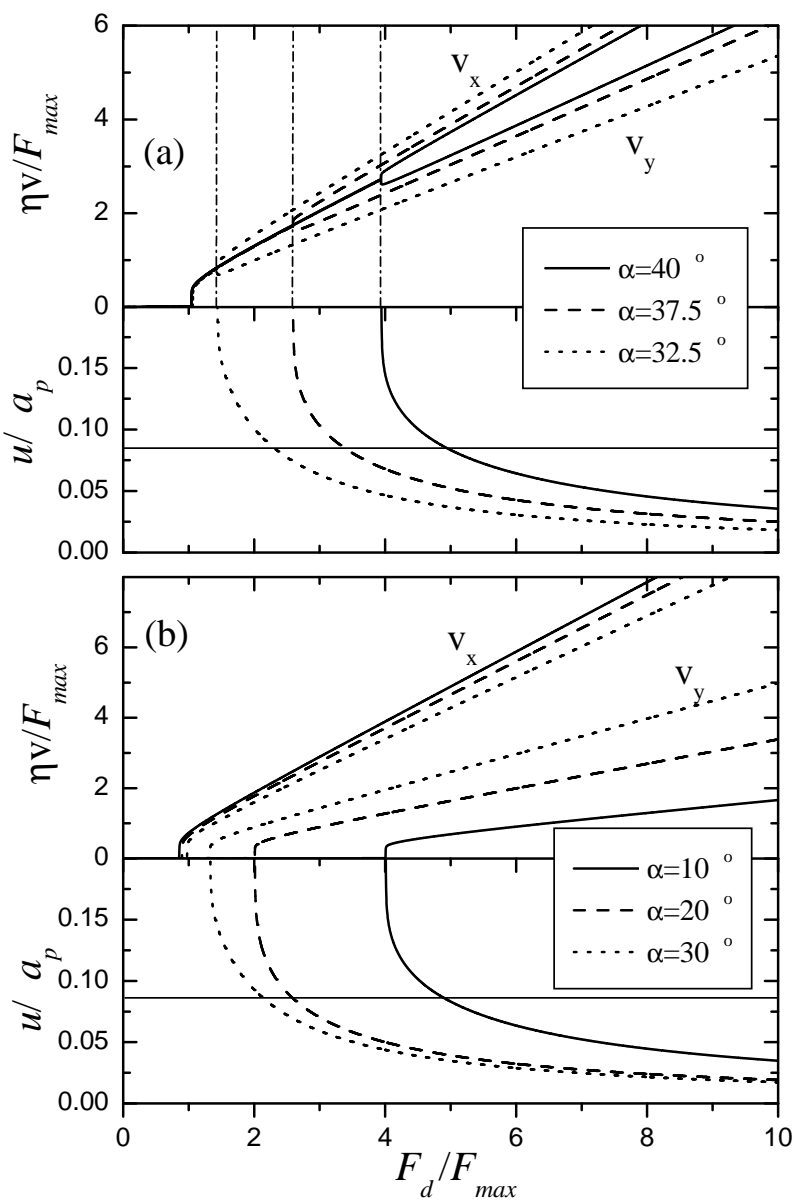

FIG. 2. Center of mass velocity components, $v_{x}$ and $v_{y}$, and root-mean-square caged vortex fluctuations, $u$, vs. driving force magnitude, $F_{d}$, for several driving force directions $\alpha$ at $T=0$. (a) $32.5^{\circ} \leq \alpha \leq 40^{\circ}$ : vortex motion is trapped in the $[1,1]$ direction $\left(v_{x}=v_{y}\right)$ at the $F_{d}$ values indicated by the vertical dot-dashed lines in the top panel. (b) $0<\alpha<32.5$ : vortex motion is trapped in the $[1,0]$ direction $\left(v_{y}=0\right)$. The horizontal lines in the bottom panels of (a) and (b) are $u=c_{L} a_{v}$ for $B=2 B_{\phi}$ and $c_{L}=0.12$ (see text), where the VL melts.

We choose to present the numerical results for the expressions derived in Sec. II as functions of the driving force $\mathbf{F}_{d}$, instead of $\mathbf{v}$. To do so we invert Eq. (114) by an iterative method. We start at a high value of the driving force magnitude, $F_{d}=50 \kappa a_{p}$, and a given orientation of $\mathbf{F}_{d}$ with respect to the $x$-axis, $\alpha$ (Fig. [1(a)), and approximate the solution by the result for infinite drive $\mathbf{v}=\mathbf{F}_{d} / \eta$. Then $F_{d}$ is decreased by small steps, keeping $\alpha$ fixed. At each step, $\mathbf{v}$ in the right hand side of Eq. (14) is replaced by the $\mathbf{v}$ obtained in the previous step. From the $\mathbf{v}$ vs. $\mathbf{F}_{d}$ relationship thus obtained, the $u$ and $T_{d m}$ vs. $F_{d}$ curves follow using Eqs. (13) and (15). The results are shown in Figs. 20. In the figures shown here the following units are used. i) Force: $F_{\max }=8 \pi U_{1} / a_{p}=$ magnitude of the maximum force exerted by the square pinning potential defined in Eqs. (17). ii) Velocities: $F_{\max } / \eta$. iii) Temperatures: $T_{m}=$ VL equilibrium melting temperature (see Sec. III).

In Fig. 2 we show $\mathbf{v}$ and $u$ as functions of $F_{d}$ for several $\alpha$ at $T=0$. The horizontal lines in the bottom panel of Figs. 2(a) and 2(b) indicate the $u=c_{L} a_{v}$ lines for a vortex density corresponding to two vortices per pin ( $\left.B=2 B_{\phi}\right)$, with $c_{L}=0.12$, at which the VL melts, according to Lindemann's criterion. The region of validity of our dynamical cage model is below this line. The $v$ vs. $F_{d}$ curves in the top panels of Figs. 2(a) and 2(b) show that the CM velocity is not parallel to the driving force, which leads to anisotropic V-I curves. This anisotropy is more clearly seen by plotting the direction of motion, $\theta$, as a function of the driving force magnitude, as shown in Fig. 3, for fixed directions of drive, $\alpha$. These plots show that, depending on $\alpha$, the moving VL is attracted towards the $[1,0]$ or $[1,1]$ directions. This behavior is similar to that seen in recent numerical simulations 5 . Above the $c_{L}=0.12$ lines in the bottom panel of Figs. 2(a) and 2(b) we find a discontinuous jump in $u$, accompanied by the trapping of moving caged vortex along $[1,0]$ for $0<\alpha<32.5^{\circ}$ [Fig. 2(b)], or along [1,1] for $32.5^{\circ}<\alpha<45^{\circ}$ [Fig. 2(a)]. This trapping is similar to transverse pinning observed in numerical simulations and JJA experiments. $\mathrm{E}^{\mathrm{O}}$ This is interesting because it indicates that, even though our dynamical cage model is not strictly valid in the region where trapping occurs, it contains physical ingredients capable of describing the phenomenon. We also note that the jump in $u$ occurs because for vortex motion along $[1,0]$ and $[1,1]$ there are terms in the sums in Eq. (13) for which $\mathbf{Q} \cdot \mathbf{v}=0$. According to the discussion in Sec. II. the perturbation expansion for $u$ breaks down and a large contribution to $u$ results from the terms in the sum in Eq. (13) with $\mathbf{Q} \cdot \mathbf{v}=0$.

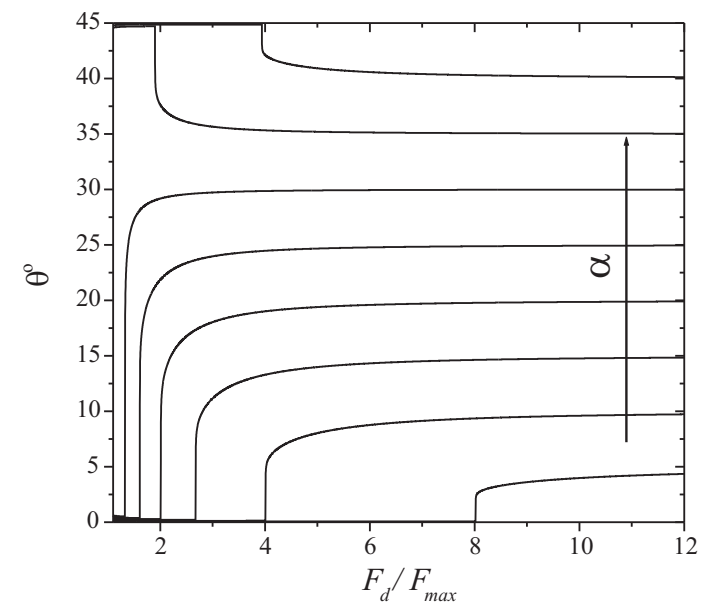

FIG. 3. Direction of motion, $\theta$, as a function of the driving force magnitude for drive directions in the range $5^{\circ} \leq \alpha \leq 40^{\circ}$ in regular increments of $5^{\circ}$. The arrow indicates increasing $\alpha$. 

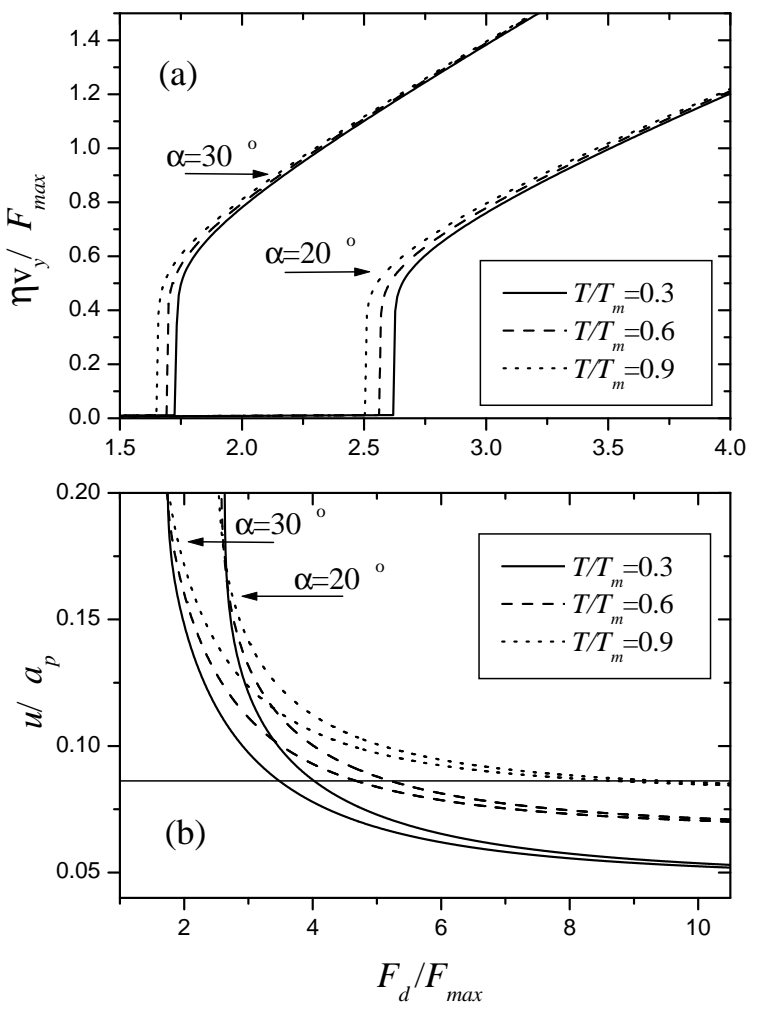

FIG. 4. Temperature dependence of: (a) center of mass velocity y-component and (b) root-mean- square displacement curves, $u$ vs. $F_{d}$.

The finite- $T$ behavior of the results is shown in Fig. 4. We find that the $\mathbf{v}$ vs. $F_{d}$ curves change little with $T$ as illustrated in Fig. 1 (a). We also find that in $u^{2}$ the $T$-dependent term in Eq. (13) gives a small contribution. This is so because the $T$-dependence in $u$ and $\mathbf{v}$ results from the third terms in the right hand side of Eqs. (13) and (14), which are, essentially, the product of two quantities which are small in the region of validity of our dynamical cage model.

The dynamical melting lines $T_{d m}$ vs. $F_{d}$ are shown in the top panel of Fig. . for several $\alpha$. In the bottom panel, the dynamical phase diagram $F_{d}$ vs $\alpha$ for $T=0$ is shown. The $F_{d}$ vs. $\alpha$ dynamical melting line in the bottom panel corresponds to the intersections of the $T_{d m}$ vs. $F_{d}$ curves shown in Fig. 5 top with the $T=0$ line. We find that for other temperatures $T<T_{m}$ the $F_{d}$ vs $\alpha$ phase diagrams are similar.

\section{CONCLUSION}

In conclusion then, we introduce a simple dynamical model for vortex lattices flowing elastically in the presence of periodic pinning. The model is solved by a perturbation method that allows the calculation of the dynamical melting curves, using Lindemann's criterion,

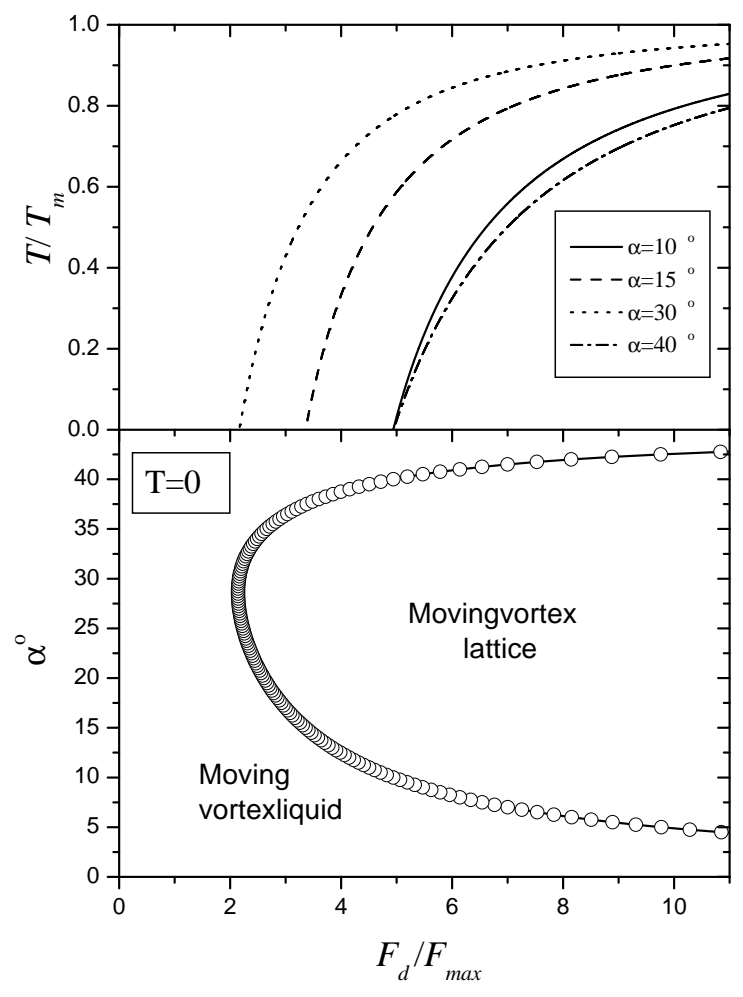

FIG. 5. Top: dynamical melting lines for $\alpha$ in the range $10^{\circ} \leq \alpha \leq 40^{\circ}$. Bottom: dynamical phase diagram $F_{d}$ vs. $\alpha$ for $T=0$.

and of the V-I curves. This approach is valid for periodic pinning potentials in general, and predicts that the moving vortex-lattice melting temperature varies significantly with the CM velocity only for strong pinning. The perturbation solution is applied to a square pinning lattice. The results show qualitative agreement with numerical simulation onest 6 . Namely: i) the predicted dynamical melting temperature approaches the thermal equilibrium one in the limit of very large center of mass velocities, and decreases with the velocity. The model predicts that this decrease is, essentially, as $v^{-2}$. ii) The center of mass velocity is not parallel to the driving force in general, leading to anisotropic V-I curves. The vortex lattice is attracted towards the $[1,0]$ or $[1,1]$ direction, depending on the direction of drive. Transverse pinning, with the vortex lattice motion restricted to the $[1,0]$ or $[1,1]$ directions, is found outside the region where the model is strictly valid.

For other periodic pinning potentials, and for strong pinning, we expect similar dynamical melting lines and anisotropic V-I curves.

It is significant that the model predicts anisotropic VI curves so closely resembling the numerical simulation ones. These curves follow from the relationship between the CM velocity and the driving force, Eq. (11). The 
only approximation used in our solution of this equation is the mean-field treatment of Langevin's equations for the whole vortex lattice. Our results for the V-I curves show that this approximation is justifiable.

The model can be also applied along the lines described here to study the effects of weak random potentials, either from material defects or from imperfections in the pinning array, on the melting lines and V-I curves. It can also be generalized to study transverse pinning more rigorously. Work along these lines is under way and will be reported elsewhere.

\section{ACKNOWLEDGMENTS}

Research supported in part by CNPq, FAPERJ, FUJB and FACEPE. We thank J. A. Aguiar and L. R. E. Cabral for stimulating discussions and useful suggestions.

\section{APPENDIX A:}

To obtain the perturbation expansion of Eq. (11) we write it, using Eq. (3), as

$$
\begin{aligned}
\eta \frac{d \mathbf{u}_{l}(t)}{d t}= & -\kappa \mathbf{u}_{l}(t)+\sum_{\mathbf{Q}} \mathbf{F}_{\mathbf{Q}}^{v-p} e^{i \omega_{\mathbf{Q}} t} \times \\
& \frac{1}{N_{v}} \sum_{j=1}^{N_{v}}\left(N_{v} \delta_{j, l}-1\right) e^{i \mathbf{Q} \cdot\left[\mathbf{R}_{j}+\mathbf{u}_{j}(t)\right]}+\boldsymbol{\Gamma}_{l},
\end{aligned}
$$

where $\mathbf{F}_{\mathbf{Q}}^{v-p} \equiv-i \mathbf{Q} U_{\mathbf{Q}}^{v-p}$ and $\omega_{\mathbf{Q}}=\mathbf{Q} \cdot \mathbf{v}$. Expanding $e^{i \mathbf{Q} \cdot \mathbf{u}_{j}}$ to first order, and carrying out the Fourier transformation in time, assuming periodic boundary conditions in the time interval $(0, \tau)$, justifiable for large $\tau$ $\left(\tau \gg a_{p} / v\right)$, we find

$$
\begin{aligned}
\mathbf{u}_{l}(\omega)= & \mathbf{u}_{l}^{T}(\omega)+\sum_{\mathbf{Q}} \frac{\mathbf{F}_{\mathbf{Q}}^{v-p}}{-i \eta \omega+\kappa} \frac{1}{N_{v}} \sum_{j=1}^{N_{v}}\left(N_{v} \delta_{j, l}-1\right) \times \\
& e^{i \mathbf{Q} \cdot \mathbf{R}_{j}}\left[\delta_{\omega,-\omega_{\mathbf{Q}}}+i \mathbf{Q} \cdot \mathbf{u}_{j}\left(\omega+\omega_{\mathbf{Q}}\right)\right]
\end{aligned}
$$

where $\mathbf{u}_{l}^{T}(\omega)$ is the thermal displacement

$$
\mathbf{u}_{l}^{T}(\omega)=\frac{\Gamma_{l}(\omega)}{-i \eta \omega+\kappa},
$$

$\Gamma_{l}(\omega)$ is the Fourier transform of the random force and $\delta_{K}$ denotes the Kronecker delta. The first order solution for $\mathbf{u}_{l}(\omega)$ is obtained by neglecting the linear term in Eq. (A2). We find

$$
\mathbf{u}_{l}^{(1)}(\omega)=\mathbf{u}_{l}^{T}(\omega)+\sum_{\mathbf{Q}} \frac{\mathbf{F}_{\mathbf{Q}}^{v-p} e^{i \mathbf{Q} \cdot \mathbf{R}_{l}}}{i \eta \omega_{\mathbf{Q}}+\kappa} \delta_{\omega,-\omega_{\mathbf{Q}}} .
$$

To derive Eq. A4 we use $\sum_{j=1}^{N_{v}} e^{i \mathbf{Q} \cdot \mathbf{R}_{j}}=\delta_{\mathbf{Q}, 0}$, since the vortex and pinning lattices are incommensurate.
The second order solution is obtained by substituting $\mathbf{u}_{j}$ in the linear term in the left hand side of Eq. (A2) by the first order solution. The result is

$$
\begin{aligned}
\mathbf{u}_{l}^{(2)}(\omega)= & \mathbf{u}_{l}^{(1)}(\omega)+\sum_{\mathbf{Q}} \frac{\mathbf{F}_{\mathbf{Q}}^{v-p}}{-i \eta \omega+\kappa} \times \\
& \frac{1}{N_{v}} \sum_{j=1}^{N_{v}}\left(N_{v} \delta_{j, l}-1\right) e^{i \mathbf{Q} \cdot \mathbf{R}_{j}} i \mathbf{Q} \cdot \mathbf{u}_{j}^{T}\left(\omega+\omega_{\mathbf{Q}}\right)+ \\
& \sum_{\mathbf{Q}, \mathbf{Q}^{\prime}} \frac{\mathbf{F}_{\mathbf{Q}}^{v-p} i \mathbf{Q} \cdot \mathbf{F}_{\mathbf{Q}}^{v-p}\left[e^{i \mathbf{K} \cdot \mathbf{R}_{l}}-\delta_{\mathbf{Q},-\mathbf{Q}^{\prime}}\right]}{(-i \eta \omega+\kappa)\left(i \eta \omega_{\mathbf{Q}^{\prime}}+\kappa\right)} \delta_{\omega,-\omega_{\mathbf{K}}},
\end{aligned}
$$

where $\omega_{\mathbf{Q}^{\prime}}=\mathbf{Q}^{\prime} \cdot \mathbf{v}, \omega_{\mathbf{K}}=\mathbf{K} \cdot \mathbf{v}$, and $\mathbf{K}=\mathbf{Q}+\mathbf{Q}^{\prime}$. With this solution, one can calculate the mean square displacement for a particular vortex $l, u_{l}^{2}=\frac{1}{\tau} \int_{0}^{\tau}\left\langle\left|\mathbf{u}_{l}(t)\right|^{2}\right\rangle$, which up to the second order in the pinning potential is expressed by:

$$
\begin{aligned}
u_{l}^{2}= & \frac{2 k_{B} T}{\kappa}+\sum_{\mathbf{Q}, \mathbf{Q}^{\prime}} \frac{\mathbf{F}_{\mathbf{Q}}^{v-p} \cdot \mathbf{F}_{\mathbf{Q}^{\prime}}^{v-p} e^{i \mathbf{K} \cdot \mathbf{R}_{l}}}{\eta^{2} \omega_{\mathbf{Q}}^{2}+\kappa^{2}} \delta_{\omega_{\mathbf{Q}},-\omega_{\mathbf{Q}^{\prime}}}+ \\
& \frac{2 k_{B} T}{\kappa} \frac{1}{N_{v}} \sum_{\mathbf{Q}} \frac{Q^{2} \mathbf{F}_{\mathbf{Q}}^{v-p} \cdot \mathbf{F}_{\mathbf{Q}^{\prime}}^{v-p}}{\eta^{2} \omega_{\mathbf{Q}}^{2}+4 \kappa^{2}} \times \\
& {\left[\left(N_{v}-2\right) e^{i \mathbf{K} \cdot \mathbf{R}_{l}}+\delta_{\mathbf{Q},-\mathbf{Q}^{\prime}}\right] }
\end{aligned}
$$

Taking the average over the $N_{v}$ vortices and assuming $N_{v} \gg 1$, Eq. 13. is derived.

A similar procedure is used to calculate the driving force dependence of $\mathbf{v}$. Expanding Eq. (7) in the form

$$
\begin{aligned}
\eta \mathbf{v}= & \mathbf{F}_{d}+\frac{1}{N_{v}} \sum_{j=1}^{N_{v}} \frac{1}{\tau} \times \\
& \int_{0}^{\tau} \sum_{\mathbf{Q}}\left\langle\mathbf{F}_{\mathbf{Q}}^{v-p} e^{i \mathbf{Q} \cdot \mathbf{R}_{j}} e^{i \omega_{\mathbf{Q}} t}\left[1+\mathbf{Q} \cdot \mathbf{u}_{j}(t)\right]\right\rangle,
\end{aligned}
$$

and using the second order solution for $u_{l}(t)$ we obtain Eq. (14).

\footnotetext{
* e-mail: gmc@if.ufrj.br

${ }^{1}$ B.N.J. Persson, Sliding Friction: Physical Principles and Applications (Springer, Heidelberger, 1998)

${ }^{2}$ R. S. Newrock, C.J. Lobb, U. Geigenmuller, and M. Octavio, Solid State Phys. 54, 263 (2000).

${ }^{3}$ A.N. Lykov, Adv. Phys. 42, 263 (1993). V.V. Moshchalkov, V. Bruyndoncx, L. Van Look, M. J. Van Bael, Y. Bruynseraede, and T. Tomomura, Handbook of Nanostructured Materials and Nanotechnology, Vol.3, H.S. Nalwa ed. (Academic Press, 2000)
} 
${ }^{4}$ G. Carneiro J. Low Temp. Phys. 117, 1323 (1999); Phys. Rev. B 62, 14661 (2000).

${ }^{5}$ G. Carneiro, Phys. Rev. B submitted.

${ }^{6}$ V.I. Marconi and D. Domínguez, Phys. Rev. Lett. 82, 4922 (1999); Phys. Rev B 63, 174509 (2001).

7 V.I. Marconi, S. Candia, P. Balenzuela, H. Pastoriza, and D. Domíngez , Phys. Rev. B 62, 4096 (2000).

${ }^{8}$ A. Schmid and W. Hauger, J. Low Temp. Phys. 11, 667 (1973).

${ }^{9}$ C. Richhardt, C.J. Olson, and F. Nori, Phys. Rev. B 58, 6534 (1998).
${ }^{10}$ C. Reichhardt and G.T. Zimányi, Phys. Rev. B 61, 14354 (2000).

11 A. Houghton, R.A. Pelcovits and A. Sudbø, Phys. Rev. B 40, 6763 (1989).

${ }^{12}$ E. Frey, D. R. Nelson and D. S. Fisher, Phys. Rev. B 49, 9723 (1994).

${ }^{13}$ D. S. Fisher, Phys. Rev. B 22, 1190 (1980).

14 A.E. Koshelev and V.M Vinokur, Phys. Rev. Lett. 73, 3580 (1994).

15 E.H. Brandt, Rep. Prog. Phys. 58, 1465 (1995).

${ }^{16}$ D. S. Fisher, Phys. Rev. B 31, 1396 (1984). 